Algebraic 83 Geometric $\mathcal{T}$ opology

Volume 5 (2005) 1419-1432

Published: 15 October 2005

ATG

\title{
Intrinsically linked graphs and even linking number
}

\author{
Thomas Fleming
}

Alexander Diesl

\begin{abstract}
We study intrinsically linked graphs where we require that every embedding of the graph contains not just a non-split link, but a link that satisfies some additional property. Examples of properties we address in this paper are: a two component link with $l k(A, L)=k 2^{r}, k \neq 0$, a non-split $n$-component link where all linking numbers are even, or an $n$-component link with components $L, A_{i}$ where $l k\left(L, A_{i}\right)=3 k, k \neq 0$. Links with other properties are considered as well.

For a given property, we prove that every embedding of a certain complete graph contains a link with that property. The size of the complete graph is determined by the property in question.
\end{abstract}

AMS Classification 57M15; 57M25,05C10

Keywords Intrinsically linked graph, spatial graph, graph embedding, linking number

\section{Introduction}

In the early 1980's, Sachs, and independently, Conway and Gordon proved that every embedding of $K_{6}$ contains two disjoint cycles that form a nontrivial link [1], 3]. Since that time there has been extensive study of intrinsically linked graphs, that is, graphs whose every embedding into three-space contains a nonsplit link. The classification of these graphs, in terms of excluded minors, was settled by Robertson, Seymour and Thomas in [10. Study of intrinsically linked graphs has turned to finding conditions that guarantee more complex structures within every embedding of the graph. For example, one could require that every embedding of a graph contains two disjoint links [2, or a link with three or more components [5] [1], or a link where one component is a nontrivial knot [7], or a two component link whose linking number is larger than some constant [4] [12].

It is this last case that interests us here. We will prove that every embedding of certain complete graphs always contains links (of various numbers of 
components) whose linking numbers are multiples of some constant (generally a power of two). These results are Ramsey theoretic in nature, in that they imply certain structures arise in sufficiently large complete graphs. In essence, we are striving to prove that given stringent requirements on a link, we can find a graph so that every embedding of that graph contains a link meeting those requirements.

In the papers of Flapan [4] and Shirai and Taniyama [12, the authors study graphs that always contain two component links with linking number greater than or equal to $k$, where here, in our investigation of two component links, we demand that the linking number be a multiple of a power of two. This is a much more restrictive condition and hence it should not be surprising that our bounds are much larger.

For two component links, we have the following theorems:

Theorem 2.5 Every embedding of $K_{\beta_{r}}$ contains a two component link with $l k(A, L)=2^{r} k$, for some $k \neq 0$.

Theorem 4.1 Every embedding of $K_{35}$ contains a link of two components with $l k(A, L)=3 k$, for some $k \neq 0$.

We can obtain a similar result for three component links, but we would like to see it strengthened so that all pairwise linking numbers are zero mod $2^{r}$.

Theorem 3.1 Every embedding of $K_{\delta_{r}}$ contains a non-split three component link $L, W, A$ with $l k(L, W)=2^{r} k, l k(L, A)=2^{r} k^{\prime}$ and $l k(W, A) \equiv 0 \bmod 2$, for some $k, k^{\prime} \neq 0$.

There are also a number of results for links of arbitrarily many components.

Corollary 2.6 Every embedding of $K_{\beta_{n, r}^{\prime}}$ contains an $n+1$ component link $L, Z_{1}, \ldots, Z_{n}$ with $l k\left(L, Z_{i}\right)=2^{r} k$, for some $k \neq 0$.

Corollary 4.2 Every embedding of $K_{7 \alpha_{3 n}^{\prime}}$ contains an $n+1$ component link $L, Z_{1}, \ldots, Z_{n}$ with $l k\left(L, Z_{i}\right)=3 k$, for some $k \neq 0$.

Theorem 3.2 Every embedding of $K_{\epsilon_{n}}$ contains a non-split link of $n+1$ components where all the pairwise linking numbers are even.

The sequence $\gamma_{n}=\Pi_{i=0}^{n-1}\left(2^{i}+1\right)$ plays a significant role in determining the size of a complete graph required for some of these properties. Unfortunately, $\gamma_{n}$ 
grows faster than $n^{n}$. Obviously, we would like to see better bounds on the number of vertices required. We also note that in this paper we provide upper bounds for the number of vertices required for these behaviors. Obvious lower bounds are seven for all cases [3], and ten for cases involving links of three or more components [6]. Finding better lower bounds or even the minimum number of vertices required for these properties remain open questions.

\section{The basic linking construction}

Our primary technique in this paper is to take two cycles that link with a third and use them to produce new cycles with various properties. To that end, the following technical lemmas will be used extensively in later results.

Lemma 2.1 Let a graph $G$ be embedded such that there exists a three component link with components $L, Z, W$ which have $l k(L, Z)=q_{1}>0$, $l k(L, W)=q_{2}>0$ with $q_{i} \equiv 0 \bmod 2^{r}$. Assume that there exist $2^{r+1}+1$ paths $P_{i}$ from $Z$ to $W$ with distinct end points such that the interior of the $P_{i}$ are disjoint from $L, W, Z$ and from the other $P_{i}$. Further assume that when traversing $W$ in the direction of its orientation, we meet the $P_{i}$ in ascending order, and when traversing $Z$ in a similar fashion, we meet them in descending order. Then this embedding of $G$ contains a two component link $A \cup L$ with $l k(A, L)=k 2^{r+1}$, for some $k \neq 0$.

Proof Clearly, if $q_{1}$ or $q_{2}$ is zero $\bmod 2^{r+1}$, we are done, so we may assume that $q_{1} \equiv q_{2} \equiv 2^{r} \bmod 2^{r+1}$. Orient the $P_{i}$ such that going from $Z$ to $W$ is the positive direction.Let $A_{1}$ be the oriented simple closed curve formed by traversing $P_{1}$ in the positive direction, along $W$ in the positive direction from $P_{1}$ to $P_{2}$, in the negative direction along $P_{2}$, and finally along $Z$ in the positive direction from $P_{2}$ to $P_{1}$. See Figure 1] Define the other $A_{i}$ similarly.

Note that now, we have that in $H_{1}\left(\mathbf{R}^{3}-L ; \mathbf{Z}\right)$;

$$
[Z]+[W]=\sum\left[A_{i}\right]
$$

Notice $[Z]+[W]=q_{1}+q_{2}$, which is $0 \bmod 2^{r+1}$ and non-zero integrally.

For $1 \leq i \leq 2^{r+1}$, let $S_{i}=\sum_{j=1}^{i}\left[A_{i}\right]$.

Suppose $S_{i} \equiv 0 \bmod 2^{r+1}$ for some $i$. If $S_{i} \neq 0$ integrally, let $A=A_{1} \cup A_{2} \cup$ $\ldots \cup A_{i}$. If $S_{i}=0$ integrally, then $\sum_{i+1}^{2^{r+1}+1}\left[A_{j}\right]=q_{1}+q_{2}$. Thus by setting $A=A_{i+1} \cup \ldots \cup A_{2^{r+1}+1}$, we have the desired result. 


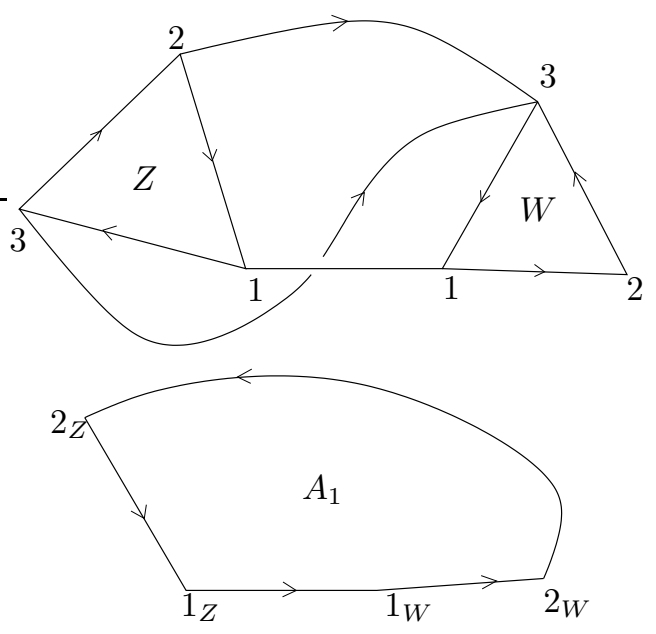

Figure 1: Constructing the $A_{i}$ for $\mathrm{r}=1$

Thus we may now assume that $S_{i} \neq 0 \bmod 2^{r+1}$ for all $i$. However, we have $2^{r+1}$ values, so for some $i<j$, we have that $S_{i} \equiv S_{j} \bmod 2^{r+1}$. Thus $0 \equiv S_{j}-S_{i} \equiv \sum_{k=i+1}^{j}\left[A_{k}\right] \bmod 2^{r+1}$. Now, as before, if $S_{j}-S_{i} \neq 0$ integrally, let $A=A_{i+1} \cup \ldots A_{j}$. If $S_{j}-S_{i}=0$ integrally, then let $A=A_{1} \cup \ldots \cup A_{i} \cup$ $A_{j+1} \cup \ldots \cup A_{2^{r+1}+1}$.

Define $\alpha_{1}=6, \alpha_{2}=10$, and for $m \geq 1, \alpha_{2 m+1}=2 \alpha_{2 m}+6$ and $\alpha_{2 m+2}=$ $2 \alpha_{2 m+1}$. Then for $m \geq 1$ we have $\alpha_{2 m+1}=6\left(\sum_{j=0}^{m} 4^{j}\right)-4^{m}$. Note that $\alpha_{1}=6 \alpha_{1}^{\prime}$ and for $n \geq 2, \alpha_{n}<6 \alpha_{n}^{\prime}$, where $\alpha_{n}^{\prime}$ is the sequence defined before Corollary 2.4.

Lemma 2.2 Every embedding of $K_{\alpha_{n}}$ contains a nonsplit link of $n+1$ components labeled $L, Z_{1}, \ldots, Z_{n}$, where $l k\left(L, Z_{i}\right) \neq 0$ for all $i$.

Proof The case $n=1$ was shown in 3 .

We will proceed by induction. The base case is $n=2$, and the desired result for $K_{10}$ was shown in [6]. The inductive step for the even and odd cases must be handled separately.

Now suppose that $K_{\alpha_{n-1}}$ contains such of link of $n$ components, where $n$ is even. The graph $K_{\alpha_{n}}$ is $K_{2 \alpha_{n-1}}$ and we may partition it into two disjoint copies of $K_{\alpha_{n-1}}$. Thus we have found two cycles, $L$ and $L^{\prime}$ which each have non-zero linking number with $n-1$ other cycles. Clearly if $l k\left(Z_{i}, L^{\prime}\right) \neq 0$ or 
$l k\left(Z_{i}^{\prime}, L\right) \neq 0$ for some $i$, we are done. So we may assume that all such linking numbers are zero.

Now, choose two vertices $l_{1}$ and $l_{2}$ in $L$ and two vertices $l_{1}^{\prime}$ and $l_{2}^{\prime}$ in $L^{\prime}$. Let $P$ be a path in $L$ connecting $l_{1}$ to $l_{2}$, and $P^{\prime}$ be a path in $L^{\prime}$ connecting $l_{1}^{\prime}$ to $l_{2}^{\prime}$. Since this is a complete graph, there are edges connecting $l_{1}$ to $l_{1}^{\prime}$ and $l_{2}$ to $l_{2}^{\prime}$. Note that the interiors of these edges are disjoint from $L, L^{\prime}, Z_{i}$, and $Z_{i}^{\prime}$. Let $V$ denote the cycle formed by these edges and $P$ and $P^{\prime}$. See Figure 2.

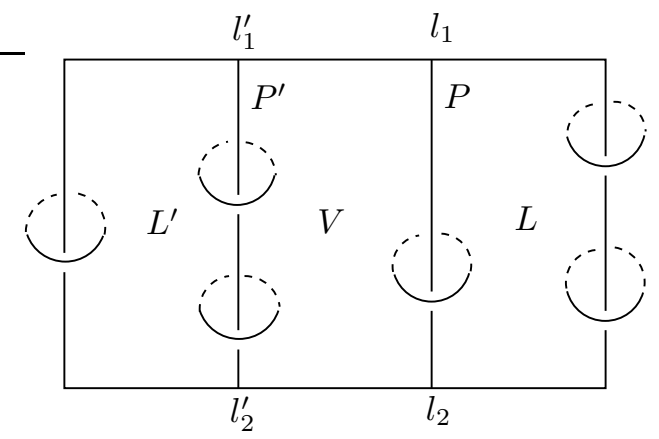

Figure 2: The cycles $L, L^{\prime}$ and $\mathrm{V}$ in the case $n=4$. The $Z_{i}$ are shown as ovals.

Now, examine $l k\left(V, Z_{i}\right)$ and $l k\left(V, Z_{i}^{\prime}\right)$. If $n$ of these are non-zero, then we have constructed the desired link. If fewer than $n-1$ of these are non-zero, then we take the cycle $L \cup V \cup L^{\prime}$, and since linking number is additive, we have the desired link once again.

If exactly $n-1$ of the $Z_{i}, Z_{i}^{\prime}$ have non-zero linking number with $V$, then as $n-1$ is odd, more than half of them came from one set, say the $Z_{i}^{\prime}$. Again using the additivity of linking number, the cycle $V \cup L$, has $l k\left(V \cup L, Z_{i}\right) \neq 0$ for at least $\frac{n}{2}$ of the $Z_{i}$, and similarly for the $Z_{i}^{\prime}$. This gives a link with $n+1$ components with the desired properties when $n$ is even.

When $n$ is odd, the inductive step requires more care. In the graph $K_{\alpha_{n}}$ we may find a two copies of $K_{\alpha_{n-1}}$ as well as a copy of $K_{6}$. The latter will tip the balance in our favor.

Again choose vertices $l_{i}$ and $l_{i}^{\prime}$. Choose also vertices $x$ and $y$ on one of the two linked triangles in $K_{6}$, call this triangle $L^{\prime \prime}$, the other $T$. Let $P$ and $P^{\prime}$ be the paths in $L$ and $L^{\prime}$ as before. Now let $V$ be the cycle formed by $P$, the edge $l_{2}-l_{2}^{\prime}, P^{\prime}, l_{1}^{\prime}-x, x-y, y-l_{1}$. See Figure 3.

Now, clearly $l k\left(Z_{i}, L^{\prime}\right), l k\left(Z_{i}^{\prime}, L\right), l k(L, T)$, and $l k\left(L^{\prime}, T\right)$ must be zero, or we have the desired link. Once again, if $n$ or more of the $Z_{i}, Z_{i}^{\prime}$ have non-zero 


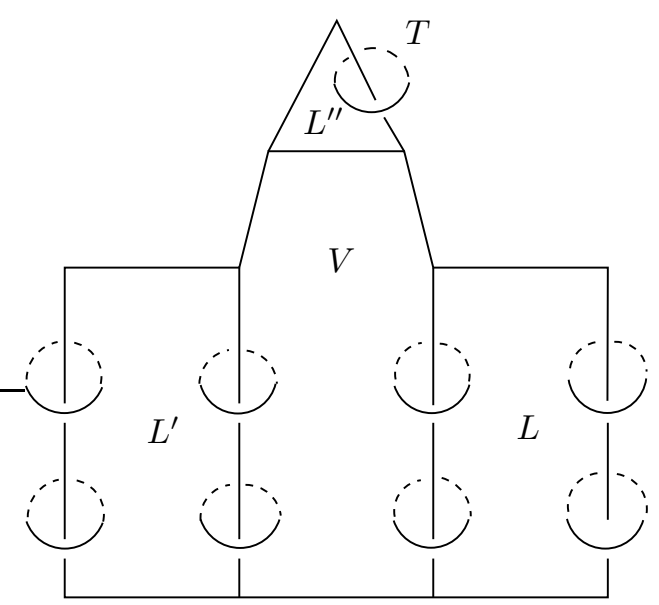

Figure 3: The cycles $L, L^{\prime}, L^{\prime \prime}$ and $\mathrm{V}$ in the case $n=5$. The $Z_{i}$ and $T$ are shown as ovals.

linking number with $V$ we are done, and if fewer than $n-1$, then we take $L \cup V \cup L^{\prime}$ as before. This leaves only the case where $n-1$ of the $Z_{i}, Z_{i}^{\prime}$ have non-zero linking number with $V$.

If $l k(T, V) \neq 0$, then take the curve $V$. This has non-zero linking number with $n-1$ of the $Z_{i}, Z_{i}^{\prime}$, and non-zero linking with $T$, so we are done in this case as well.

Thus, we may assume $l k(T, V)$ is zero. Let $C$ be the subset of $\left\{Z_{i}, Z_{i}^{\prime}\right\}$ which have zero linking number with $V$, and $D$ be the subset which have non-zero linking number with $V$. Suppose $r$ elements of $C$ and $s$ elements of $D$ have non-zero linking with $L^{\prime \prime}$. Of these $s$ elements, suppose $t$ satisfy the equation $l k\left(Z_{i}, L\right)+l k\left(Z_{i}, V\right)+l k\left(Z_{i}, L^{\prime \prime}\right)=0$ (or the similar version for $\left.Z_{i}^{\prime}, L^{\prime}\right)$. Then at least $n+r-s+t$ elements have non-zero linking with $V \cup L^{\prime \prime}$ and at least $n-r+s-t$ have non-zero linking with $V \cup L \cup L^{\prime} \cup L^{\prime \prime}$. Clearly one of these is greater than or equal to $n$, so we are done.

Lemma 2.3 Every embedding of $K_{\alpha_{n}}$ contains a nonsplit link of $n+1$ components labeled $L, Z_{1}, \ldots, Z_{n}$, where $l k\left(L, Z_{i}\right) \equiv 1 \bmod 2$ for all $i$.

The proof of Lemma 2.3 proceeds exactly as for Lemma 2.2 replacing the phrase (non-)zero with (non-)zero mod two.

Let $\alpha_{1}^{\prime}=1, \alpha_{2 m}^{\prime}=2 \alpha_{2 m-1}^{\prime}$, and $\alpha_{2 m+1}^{\prime}=2 \alpha_{2 m}^{\prime}+1$. Again, a calculation gives $\alpha_{2 m-1}^{\prime}=\frac{4^{m}-1}{3}$. 
Corollary 2.4 If every embedding of $G$ contains a two component link with non-zero linking number, then every embedding of $*^{\alpha_{n}^{\prime}} G$ contains an $n+1$ component link $L, Z_{i}$ with $l k\left(L, Z_{i}\right) \neq 0$.

Proof Note that the sequence $\alpha_{n}^{\prime}$ satisfies the following recursive relations: $\alpha_{n}^{\prime}=2 \alpha_{n-1}^{\prime}$ if $n$ is even, $\alpha_{n}^{\prime}=2 \alpha_{n-1}^{\prime}+1$ if $n$ is odd, and $\alpha_{1}^{\prime}=1$.

The proof is now the same as the proof of Lemma 2.2. but replace $K_{10}$ with $G * G$ and $K_{6}$ with $G$.

Naturally, for Lemma 2.3 we have a corollary similar to Corollary 2.4 ,

We will now obtain a sequence of links that can be thought of as the "mod two Whitehead links."

Let $\beta_{0}=6, \beta_{1}=10$ and $\beta_{r}=\alpha_{2^{r}}^{\prime}\left(\gamma_{r}+3\right)$ for higher $r$. Here $\gamma_{r}=\Pi_{i=0}^{r-1}\left(2^{i}+1\right)$.

Theorem 2.5 Every embedding of $K_{\beta_{r}}$ contains a two component link with $l k(A, L)=2^{r} k$, for some $k \neq 0$.

Proof Notice that the case $r=0$ was proved for $K_{6}$ in [3].

The case $r=1$ is direct. By [6] every embedding of $K_{10}$ contains a three component link, with $l k(L, Z)=q_{1}, l k(L, W)=q_{2}$ with $q_{i}$ odd. Choose an orientation of $L \cup W \cup Z$ so that the $q_{i}$ are positive. Clearly $Z$ and $W$ contain at least three vertices. Since $K_{10}$ is a complete graph, we have edges from each vertex of $Z$ to all vertices of $W$, and the interiors of these edges are disjoint from $L, W, Z$. We may choose $P_{i}$ from among these edges that satisfy the conditions of Lemma 2.1, so we can find the desired link.

We will now give the construction for general $r$.

Every embedding of $K_{m+3}$ contains a link composed of a triangle and an $m$ cycle with nonzero linking number by [9]. By Corollary 2.4, we know that $K_{m+3} * K_{m+3}$ contains a three component link where at least two of the three pairwise linking numbers are nonzero. Choosing the triangles to act as $L$, we know that the $Z_{i}$ have $m$ vertices.

Thus, in $K_{2 m+6}$ we may find a three component link $L, Z_{1}, Z_{2}$ with $l k\left(L, Z_{i}\right) \neq$ 0 , and with each $Z_{i}$ containing $m$ vertices. Now, by Corollary 2.4 and the preceding discussion, if we want a $n+1$ component link with these properties, we need only take $K_{\alpha_{n}^{\prime}(m+3)}$. 
Suppose $n$ is a large power of two, and $m$ is large. Pair up the $Z_{i}$. If $l k\left(Z_{i}, L\right)$ is even for some $i$, ignore that pair. For the remaining pairs (where $l k\left(Z_{i}, L\right) \equiv 1$ mod 2) choose three evenly spaced paths between the members of that pair. Applying Lemma 2.1 to these pairs, we have new cycles $A_{i}$ with $l k\left(L, A_{i}\right)=2 k$ $(k \neq 0)$. Now including the $Z_{i}$ with even linking number, we have $\frac{n}{2}$ cycles which have nonzero even linking number with $L$ and each cycle has at least $2\left(\frac{m}{3}+1\right)$ vertices. As long as we have chosen $m$ and $n$ large enough, we may iterate this process to obtain the desired link.That is, suppose $l k\left(Z_{1}, L\right) \equiv$ $l k\left(Z_{2}, L\right) \equiv 0 \bmod 2^{s-1}$ but are non-zero integrally, and that the $Z_{i}$ have at least $m^{\prime}$ vertices. If $l k\left(Z_{i}, L\right) \equiv 0 \bmod 2^{s}$, we are done. So we may assume that $l k\left(Z_{1}, L\right) \equiv l k\left(Z_{2}, L\right) \equiv 2^{s-1} \bmod 2^{s}$. Choosing $2^{s}+1$ paths from $Z_{1}$ to $Z_{2}$ allows us to apply Lemma 2.1 to obtain a cycle $Z$ with $l k(Z, L)=k 2^{s}, k \neq 0$, such that $Z$ has at least $2\left(\frac{m^{\prime}}{2^{s}+1}+1\right)$ vertices.

Thus, if we want a two component link with $l k(A, L) \equiv 0 \bmod 2^{r}$, we choose $n=2^{r}$, and $m=\gamma_{r}$. Clearly we need $n=2^{r}$ components linked with $L$, as after each pairing, we have increased the linking number of $Z_{i}$ with $L$ by a power of two, but have halved the number of components. However, on iteration $s$ in this procedure (moving from $2^{s-1}$ to $2^{s}$ ) we need to have at least $2^{s}+1$ vertices in each $Z_{i}$ to successfully apply Lemma 2.1. Since the number of vertices is strictly decreasing in this process, it suffices to guarantee that we have $c_{r}$ vertices at the $r$ th iteration, where $c_{r} \geq 2^{r}+1$. If we have $c_{s}$ vertices at the beginning of iteration $s$, we will be left with $c_{s+1}=2\left(\frac{c_{s}}{2^{s}+1}+1\right)$ vertices at the beginning of iteration $s+1$. Thus, when $c_{s+1}$ is even, we have $c_{s}=\left(2^{s}+1\right)\left(\frac{c_{s+1}}{2}-1\right)$, and we want to choose $m \geq c_{1}$. Choosing $c_{r}=2^{r}+2$ gives $c_{r-1}=\left(2^{r-1}+1\right)\left(2^{r-1}\right)$. Since we are looking only for a bound, let us take $c_{r-1}=\left(2^{r-1}+1\right)\left(2^{r-1}\right)+2$. This gives $c_{r-2}=\left(2^{r-2}+1\right)\left(2^{r-1}+1\right)\left(2^{r-2}\right)$. Continuing this process gives $c_{1}=\gamma_{r}$. Thus, by taking $m=\gamma_{r}$ we ensure that at each stage, we have a sufficient number of vertices to apply Lemma 2.1.

We have calculated the actual number of vertices required for some small cases, and $\gamma_{r}$ provides a very reasonable estimate. Denoting the required number of vertices $v_{r}$, we note that $\gamma_{1}=v_{1}=3 ; \gamma_{2}=v_{2}=6 ; \gamma_{3}=30, v_{3}=27 ; \gamma_{4}=$ $270, v_{4}=261$.

Let $\beta_{n, r}^{\prime}=\alpha_{n 2^{r}}^{\prime}\left(\gamma_{r}+3\right)$.

Corollary 2.6 Every embedding of $K_{\beta_{n, r}^{\prime}}$ contains an $n+1$ component link $L, Z_{1}, \ldots, Z_{n}$ with $l k\left(L, Z_{i}\right)=2^{r} k$, for some $k \neq 0$.

Proof By Lemma2.2 we can find an $n 2^{r}+1$ component link where $l k\left(Z_{i}^{\prime}, L\right) \neq$ 0 and each of the $Z_{i}$ has at least $\gamma_{r}$ vertices. Applying the construction of 
Theorem 2.5 to each set of $2^{r}$ components, we obtain the desired link. Note that the construction of one such $Z_{i}$ does not affect $L$ or the other $Z_{i}$ so we may treat them independently.

Note that Theorem 2.5] implies that every embedding of $K_{10}$ contains a nonsplit two component link with even linking number. In fact, we can improve upon that result.

Proposition 2.7 Every embedding of $K_{10}$ contains a two component link $A, L$ with $l k(A, L) \equiv 2 \bmod 4$.

Proof The proof of Thoerem 2.4 in [8] shows that every embedding of $K_{10}$ contains a two component link with odd unoriented Sato-Levine invariant. A pair of intersection points of opposite sign contribute an even quantity to $\beta^{*}$; a pair of intersection points with matching sign contribute an odd amount. Thus, it is easy to see that $\beta^{*}(A, L)$ is even if and only if $l k(A, L) \equiv 0 \bmod 4$. Since the constructed link has odd unoriented Sato-Levine invariant, it must have linking number $2 \bmod 4$.

\section{Multiple component constructions}

We would like to see the following theorem extended to have all three pairwise linking numbers be zero mod $2^{r}$.

Let $\delta_{r}=\alpha_{3\left(2^{r}\right)}^{\prime}\left(\left(2^{2 r-1}+2^{r}\right) \gamma_{r}+3\right)$.

Theorem 3.1 Every embedding of $K_{\delta_{r}}$ contains a non-split three component link $L, W, A$ with $l k(L, W)=2^{r} k, l k(L, A)=2^{r} k^{\prime}$ and $l k(W, A) \equiv 0 \bmod 2$, for some $k, k^{\prime} \neq 0$.

Proof By Corollary 2.6, we may find a four component link $L, W, Z_{1}, Z_{2}$, with $l k(L, W) \equiv l k\left(L, Z_{i}\right) \equiv 0 \bmod 2^{r}$, but nonzero integrally. In building up the $Z_{i}$ as we did in the proof of Theorem 2.5] at each stage the linking number is multiplied by two, but the number of vertices is reduced from $c_{j}$ to $c_{j+1}$. If we had begun with $\gamma_{r}$ vertices in each cycle, the construction would end with the $Z_{i}$ containing at least four vertices. However, we will require $\left(2^{r}+1\right)^{2}$ vertices, so we multiply $\gamma_{r}$ by the smallest constant that ensures this result. Thus, each of $W, Z_{i}$ has at least $2\left(2^{2 r-1}+2^{r}+1\right)>\left(2^{r}+1\right)^{2}$ vertices. This number of vertices is necessary to form a cycle $A$ from the $Z_{i}$ so that the 
three component link $L, W, A$ will have the desired property. This number of vertices is not necessary in $W$, but unfortunately Lemma 2.2 does not allow us to control the size of the components independently.

If $l k\left(Z_{i}, W\right) \equiv 0 \bmod 2$ we are done, so suppose that $l k\left(Z_{i}, W\right) \equiv 1 \bmod$ 2. The $Z_{i}$ have $\left(2^{r}+1\right)^{2}$ vertices by construction. Find $\left(2^{r}+1\right)^{2}$ paths $P_{i}$ and construct cycles $A_{i}^{\prime}$ as in the proof of Lemma 2.1. Now, write these in a list. Group the first $2^{r}$ cycles, then leave one, then group the next $2^{r}$ cycles, etc. Look at the first $2^{r}$ cycles in $H_{1}\left(\mathbf{R}^{3}-L ; \mathbf{Z}\right)$; we know that there is some subsequence whose sum is $0 \bmod 2^{r}$. Glue these cycles together. Look at the next block of $2^{r}$ cycles. Find its subsequence. Now identify all cycles between these two. The result will be a sequence of cycles $A_{i}^{\prime}$ where $\left[A_{i}^{\prime}\right] \equiv 0 \bmod 2^{r}$ for $i$ odd, and takes arbitrary values for $i$ even. Any cycles coming before $A_{1}^{\prime}$ may be pushed around to the end of the sequence since it is cyclic.

The result is a list of $2\left(2^{r}+1\right)$ cycles, half of which are zero mod $2^{r}$. Now, ignoring the zeros, there is a list of $2^{r}+1$ numbers, and as before there must be a proper consecutive subsequence that is zero $\bmod 2^{r}$. Let $A_{2}^{\prime \prime}$ be the cycle which corresponds to this sequence, $A_{1}^{\prime \prime}$ be the cycle which is $0 \bmod 2^{r}$ that immediately precedes it, and $A_{3}^{\prime \prime}$ be the complement of these two cycles. Since $\sum\left[A_{i}^{\prime \prime}\right]_{L}=\left[Z_{1}\right]_{L}+\left[Z_{2}\right]_{L}=k 2^{r}, k \neq 0$, we know $\left[A_{3}^{\prime \prime}\right]_{L} \equiv 0 \bmod 2^{r}$.

Now examine $\left[A_{i}^{\prime \prime}\right] \in H_{1}\left(\mathbf{R}^{3}-W ; \mathbf{Z}\right)$. Again, there is a subsequence of these that sums to zero mod 2. Call the corresponding cycle $A$. Since $\sum\left[A_{i}^{\prime \prime}\right]_{W}=$ $\left[Z_{1}\right]_{W}+\left[Z_{2}\right]_{W} \equiv 0 \bmod 2$, the complement of $A$ is also zero $\bmod 2$. Call it $A^{\prime}$.

By construction, $l k(A, L) \equiv 0 \bmod 2^{r}$ and $l k(A, W) \equiv l k\left(A^{\prime}, W\right) \equiv 0 \bmod$ 2. If $l k(A, L)=0$, then as $[A]_{L}+\left[A^{\prime}\right]_{L}=\sum\left[A_{i}^{\prime \prime}\right]_{L}$, we have that $l k\left(A^{\prime}, L\right)=$ $k 2^{r}, k \neq 0$ and we are done.

Let $\epsilon_{1}=10$ and $\epsilon_{n}=\alpha_{2^{n+1}-2}^{\prime}\left(\gamma_{n}^{\prime}+3\right)$. Here $\gamma_{n}^{\prime}=\Pi_{i=1}^{n} 3\left(2^{i-1}\right)=3^{n} 2^{\frac{n(n-1)}{2}}$.

Then extending our construction yet again, we can show the following.

Theorem 3.2 Every embedding of $K_{\epsilon_{n}}$ contains a non-split link of $n+1$ components where all the pairwise linking numbers are even.

Proof The case $n=1$ is proved in Lemma 2.1 and $n=2$ is proved in Theorem 3.1. For larger $n$, we may think of $K_{\epsilon_{n}}$ as the star of $\alpha_{2^{n+1}-2}^{\prime}$ copies of $K_{\gamma_{n}^{\prime}+3}$, and so it contains a link of $2^{n+1}-1$ components, labeled $L, Z_{i}$, where $l k\left(L, Z_{i}\right) \neq 0$ and the $Z_{i}$ have $\gamma_{n}^{\prime}$ vertices. Since $2^{n+1}-2=\sum_{i=1}^{n} 2^{i}$, we may break the $Z_{i}$ into sets of size $2^{i}$ for each $1 \leq i \leq n$. 
Take $Z_{1}$ and $Z_{2}$. If $l k\left(Z_{i}, L\right) \equiv 0 \bmod 2$, label that cycle $V_{1}$ and move on. If $l k\left(Z_{i}, L\right) \equiv 1 \bmod 2$ for $i=1,2$, choosing three paths between them and forming $A_{i}$ as usual, we may produce $V_{1}$, which has $l k\left(V_{1}, L\right) \equiv 2 k_{1}$, with $k_{1} \neq 0$.

Take $Z_{3}, Z_{4}, Z_{5}, Z_{6}$. Pair these and construct $Z_{3}^{\prime}, Z_{4}^{\prime}$ which have even (and positive) linking number with $L$ as we just did for $Z_{1}$ and $Z_{2}$. If $l k\left(Z_{i}^{\prime}, V_{1}\right) \equiv 0$ mod 2, label it $V_{2}$ and continue to the next set of $Z_{i}$. If not, choose six paths from $Z_{3}^{\prime}$ to $Z_{4}^{\prime}$ and construct $A_{1}, \ldots, A_{6}$. Examine these cycles in $H_{1}\left(\mathbf{R}^{3}-\right.$ $L ; \mathbf{Z} / \mathbf{2})$. Since $l k\left(Z_{i}^{\prime}, L\right) \equiv 0 \bmod 2$ this is a binary sequence which sums to zero. Cut this sequence into the maximum number of blocks, where the sum in each block is zero. Since we can cyclicly permute the entries, there must be at least three such blocks. Produce the cycles $A_{1}^{\prime}, A_{2}^{\prime}, A_{3}^{\prime}$ by fusing the cycles in each block. Look at the $A_{s}^{\prime}$ in $H_{1}\left(\mathbf{R}^{3}-V_{1} ; \mathbf{Z} / \mathbf{2}\right)$. There is a proper subsequence whose sum is zero mod two. Call the union of those cycles $A$, and their complement $A^{\prime}$. Now, $l k\left(A, V_{1}\right) \equiv l k\left(A^{\prime}, V_{1}\right) \equiv 0 \bmod 2$. If $l k(A, L)=0$, then as $[A]_{L}+\left[A^{\prime}\right]_{L}=\left[Z_{3}^{\prime}\right]+\left[Z_{4}^{\prime}\right]$, we have that $l k\left(A^{\prime}, L\right)=2 k_{2}, k_{2} \neq 0$. Call this cycle $V_{2}$.

Take $Z_{\left(\sum_{s=1}^{j-1} 2^{s}\right)+1} \cdots Z_{\sum_{s=1}^{j} 2^{s}}$. Pair them to produce $Z_{i}^{(1)}$ that have even, nonzero linking number with $L$ as we did for $Z_{1}$ and $Z_{2}$. Now, pair the $Z_{i}^{(1)}$ and produce $Z_{i}^{(2)}$ that have $l k\left(Z_{i}^{(2)}, V_{1}\right) \equiv 0 \bmod 2$, and $l k\left(Z_{i}^{(2)}, L\right)=k 2, k \neq 0$ as we did for $Z_{3}, Z_{4}, Z_{5}, Z_{6}$. Now, pair the $Z_{i}^{(2)}$. If either member of a pair has $l k\left(Z_{i}^{(2)}, V_{2}\right) \equiv 0 \bmod 2$, it advances to the next round. Otherwise, choose twelve paths connecting the members of each pair and produce $A_{1} \ldots A_{12}$. Examine these in $H_{1}\left(\mathbf{R}^{3}-L ; \mathbf{Z} / \mathbf{2}\right)$, then $H_{1}\left(\mathbf{R}^{3}-V_{1} ; \mathbf{Z} / \mathbf{2}\right)$, breaking it into the maximum number of blocks each time. We will be left with at least three cycles that we examine in $H_{1}\left(\mathbf{R}^{3}-V_{2} ; \mathbf{Z} / \mathbf{2}\right)$, allowing us to construct $Z_{i}^{(3)}$.

Continue the process, connecting the paired cycles by $3\left(2^{k-1}\right)$ paths in the $k$ th step and producing the $A_{s}$. We have chosen our cycles $Z_{i}$ to have $\gamma_{n}^{\prime}$ vertices, which ensures that we may find these paths in up to $n$ iterative steps. Examine these $A_{s}$ in $H_{1}\left(\mathbf{R}^{3}-L ; \mathbf{Z} / \mathbf{2}\right)$, then $H_{1}\left(\mathbf{R}^{3}-V_{i} ; \mathbf{Z} / \mathbf{2}\right)$ for $i<k-1$ fusing cycles as necessary each time. We began with $3\left(2^{k-1}\right)$ cycles labeled $A_{s}$, so there must be at least three distinct cycles left after fusing $k-1$ times. Examine these three cycles in $H_{1}\left(\mathbf{R}^{3}-V_{k-1} ; \mathbf{Z} / \mathbf{2}\right)$. There is a subsequence that sums to zero, we take the corresponding cycle $W_{1}$ and its complement $W_{2}$. Now, by construction, $l k\left(W_{l}, V_{i}\right) \equiv 0 \bmod 2$ for $i<k$, and at least one of $l k\left(W_{l}, L\right)$ is even and nonzero. Choose that $W_{l}$ to be $Z_{i}^{(k)}$.

Since we began with $2^{j}$ cycles, we may repeat this process $j$ times until we 
have $V_{j}$, a cycle with $l k\left(V_{j}, V_{i}\right) \equiv 0 \bmod 2$ for $i<j$ and $l k\left(V_{j}, L\right)=2 k_{j}$ with $k_{j} \neq 0$.

Iterating this process produces the desired link.

\section{Mod 3 constructions}

It is unclear if similar constructions will work modulo integers other than 2 . We provide a first step in that direction.

Theorem 4.1 Every embedding of $K_{35}$ contains a link of two components with $l k(A, L)=3 k, k \neq 0$.

Proof The graph $K_{35}$ has $*^{5} K_{7}$ as a subgraph. By Corollary 2.4 and the discussion in the proof of Theorem 2.5 every embedding of $K_{35}$ contains a four component link $L, Z_{1}, Z_{2}, Z_{3}$ with $l k\left(L, Z_{i}\right) \neq 0$, and the $Z_{i}$ each have four vertices. Orient the $Z_{i}$ and $L$ so that $l k\left(L, Z_{i}\right)=q_{i}>0$.

If $l k\left(L, Z_{i}\right) \equiv 0 \bmod 3$ for some $i$, then we are done.

If $l k\left(L, Z_{i}\right) \equiv 1 \bmod 3$ and $l k\left(L, Z_{j}\right) \equiv 2 \bmod 3$, then we may construct four cycles $A_{i}$ as in Lemma 2.1. Here $q_{1}+q_{2} \equiv 0 \bmod 3$, and taking $S_{1}, S_{2}, S_{3}$ as before, the construction of $A$ proceeds in exactly the same manner.

Thus, we may assume that $l k\left(L, Z_{1}\right) \equiv l k\left(L, Z_{2}\right) \equiv l k\left(L, Z_{3}\right) \bmod 3$.

We now construct cycles $A_{1}, A_{2}, A_{3}, A_{4}, A_{5}$ as in Figure 4 . Note that in $H_{1}\left(\mathbf{R}^{3}-\right.$ $L ; \mathbf{Z})$

$$
\sum\left[Z_{i}\right]=\sum\left[A_{i}\right]=q_{1}+q_{2}+q_{3} \equiv 0 \bmod 3
$$

If $\left[A_{i}\right] \equiv 0$ for some $i \geq 4$, then we take $A=A_{i}$ if $\left[A_{i}\right] \neq 0$. If $\left[A_{i}\right]=0$ integrally, then let $A=\cup_{j \neq i} A_{j}$.

Suppose that $\left[A_{i}\right] \equiv 0$ for some $i \leq 3$. Again, take $A=A_{i}$ if $\left[A_{i}\right] \neq 0$ integrally. If $\left[A_{i}\right]=0$, look at the remaining $A_{j}$. We have four contiguous cycles whose sum is zero mod 3, see Figure 4. Forming the partial sums $S_{1}, S_{2}$, and $S_{3}$, either two of these are equal, or one is zero, and we may proceed as in Lemma 2.1 .

Now, if $\left[A_{i}\right] \neq 0 \bmod 3$ for all $i$, then two of $\left[A_{1}\right],\left[A_{2}\right],\left[A_{3}\right]$ are equal. Say $\left[A_{1}\right]=\left[A_{2}\right]$. Take the following sums. Let $S_{1}=\left[A_{4}\right], S_{2}=\left[A_{4}\right]+\left[A_{1}\right], S_{3}=$ $\left[A_{4}\right]+\left[A_{1}\right]+\left[A_{5}\right]$, all taken mod 3 . We can ignore the cases $S_{1} \equiv 0, S_{1} \equiv S_{2}$ and 


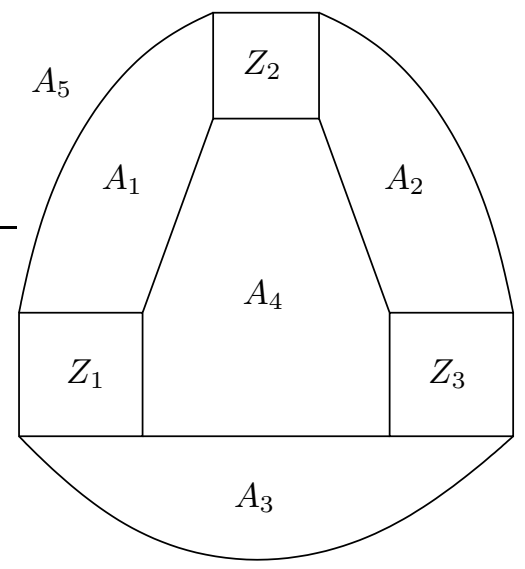

Figure 4: The cycles $A_{i}$ when using all the $Z_{i}$. The $A_{i}$ (except $A_{5}$ ) are oriented counter-clockwise, $A_{5}$ and the $Z_{i}$ clockwise.

$S_{2} \equiv S_{3}$ as each of these would imply that some $\left[A_{i}\right]$ was zero. Now, if $S_{1}=S_{3}$, we take the cycle representing their difference, and its complement. Since both cycles are connected, one of the two must satisfy the desired conditions. If $S_{2}$ is zero mod 3, the same argument applies. The hard case is when $S_{3}=0$ integrally.

The cycle corresponding to $S_{3}$ is connected, but its complement, $A_{2} \cup A_{3}$ is not. Since $S_{3}=0$, we know that $\left[A_{1}\right]=-\left(\left[A_{4}\right]+\left[A_{5}\right]\right)$. Now, since $\left[A_{i}\right]$ is non-zero $\bmod 3$ for all $i,\left[A_{4}\right] \equiv\left[A_{5}\right] \bmod 3$ (otherwise $\left.\left[A_{1}\right] \equiv 0\right)$. But now $\left[A_{1}\right] \equiv-2\left[A_{4}\right] \equiv\left[A_{4}\right] \bmod 3$.

Thus $\left[A_{1}\right] \equiv\left[A_{2}\right] \equiv\left[A_{4}\right] \equiv\left[A_{5}\right] \equiv \sum_{i \neq 3}\left[A_{i}\right] \bmod 3$. So, as $\sum\left[A_{i}\right] \equiv 0 \bmod 3$, $\left[A_{3}\right]+\left[A_{5}\right] \equiv 0 \bmod 3$. Now both $A_{3} \cup A_{5}$ and its complement $A_{1} \cup A_{2} \cup A_{4}$ are connected, so one of the two is the desired cycle $A$.

Corollary 4.2 Every embedding of $K_{7 \alpha_{3 n}^{\prime}}$ contains an $n+1$ component link $L, Z_{1}, \ldots, Z_{n}$ with $l k\left(L, Z_{i}\right)=3 k, k \neq 0$.

Proof The proof is essentially the same as the proof of Corollary 2.6

We can think of $K_{7 \alpha_{3 n}^{\prime}}$ as $\alpha_{3 n}^{\prime}$ copies of $K_{7}$. By Corollary 2.4 we have a $3 n+1$ component link $L, Z_{i}$ with $l k\left(L, Z_{i}\right) \neq 0$. The $Z_{i}$ have at least four vertices each, so breaking them into groups of three, we may now apply construction from the proof of Theorem 4.1 to each group individually to obtain the desired link. 


\section{References}

[1] G Bowlin, J Foisy, Some new intrinsically 3-linked graphs, J. Knot Theory Ramifications 13 (2004) 1021-1027 MathReview

[2] S Chan, A Dochtermann, J Foisy, J Hespen, E Kunz, T Lalonde, Q Loney, K Sharrow, $\mathbf{N}$ Thomas, Graphs with disjoint links in every spatial embedding, J. Knot Theory Ramifications 13 (2004) 737-748 MathReview

[3] J H Conway, C M Gordon, Knots and links in spatial graphs, J. Graph Theory 7 (1983) 445-453 MathReview

[4] E Flapan, Intrinsic knotting and linking of complete graphs, Algebr. Geom. Topol. 2 (2002) 371-380 MathReview

[5] E Flapan, J Pommersheim, J Foisy, R Naimi, Intrinsically n-linked graphs, J. Knot Theory Ramifications 10 (2001) 1143-1154 MathReview

[6] E Flapan, R Naimi, J Pommersheim, Intrinsically triple linked complete graphs, Topology Appl. 115 (2001) 239-246 MathReview

[7] T Fleming, Intrinsically linked graphs with knotted components, preprint

[8] T Fleming, J Pommersheim Intrinsically linked graphs and the Sato-Levine invariant, preprint

[9] B Johnson, W Johnson, On the size of links in $K_{n, n}, K_{n, n, 1}$, and $K_{n}$, J. Knot Theory Ramifications 11 (2002) 145-151 MathReview

[10] N Robertson, P Seymour, R Thomas, Sachs' linkless embedding conjecture, J. Combin. Theory Ser. B 64 (1995) 185-227 MathReview

[11] H Sachs, On spatial representations of finite graphs, from: "Finite and infinite sets, Vol. I, II (Eger, 1981)", Colloq. Math. Soc. János Bolyai 37, North-Holland, Amsterdam (1984) 649-662 MathReview

[12] M Shirai, K Taniyama, A large complete graph in a space contains a link with large link invariant, J. Knot Theory Ramifications 12 (2003) 915-919 MathReview

University of California San Diego, Department of Mathematics

9500 Gilman Drive, La Jolla, CA 92093-0112, USA

and

University of California Berkeley, Department of Mathematics

970 Evans Hall, Berkeley, CA 94720-3840, USA

Email: tfleming@math.ucsd.edu and adiesl@math.berkeley.edu

Received: 22 April 2004 Revised: 13 September 2005 